\title{
Work-wear pattern design to accommodate different working postures
}

Sara Bragança ${ }^{1}$, Miguel Carvalho ${ }^{2}$, Pedro Arezes ${ }^{1}$, Susan P. Ashdown ${ }^{3}$

${ }^{1}$ Department of Production and Systems, University of Minho, 4800-019, Guimarães, Portugal

${ }^{2}$ Department of Textile Engineering, University of Minho, 4800-019, Guimarães, Portugal

${ }^{3}$ Department of Fiber Science \& Apparel Design, Cornell University, Ithaca, NY, USA

\begin{abstract}
:
Purpose: This paper presents an analysis of several issues that are preponderant for the work-wear design. The purpose of this was to create a prototype of a women's base upper body garment, based on the information gathered.

Design/methodology/approach: All the necessary information was collected through questionnaires, anthropometric measurements and evaluation of compression forces between the different prototypes.

Findings: It was possible to conclude that to create a better design some alterations need to be made in the standard base pattern design, such as measurements across the back length.

Research limitations/implications: Despite the fact that these design changes have a great impact on the stretch ability and on the compression forces, using softer fabric, such as cotton, is always for an increased comfort. However, in more professional situation where these fabrics should not be used these design changes can really make a difference.

Practical implications: A test with a set of compression sensors showed that the simple alteration of one measurement in the design of the base patterns highly reduces the compression forces.

Social implications: These simple alterations allow the garments to adjust to the users' needs, promoting higher levels of comfort and lower levels of limitations of movement.

Originality/value: The designs presented in this paper can be easily adapted to a variety of garments, such as jackets or dresses, both for leisure or professional activities.
\end{abstract}

Keywords: work-wear; anthropometry; design; comfort; compression; stretch ability

\section{Introduction}

Clothing may cause some discomfort when different movements are made or when dynamic postures are assumed. Body movement causes its dimensions to change, for example there is an increase of the length on one side of a bending joint and the decrease of the length on the opposite side (Kirk \& Ibrahim 1966). The fact that some clothing does not accommodate the body variations in dimension over a bending joint will restrict movements and cause discomfort. The impact of the body changes in the clothing fit and comfort has already been evaluated by some authors. Choi and Ashdown (2011) evaluate the differences in body measurements between standing and siting postures, concluding that when in the sitting posture there was an increase of waist girth by $8 \%$; an 
increase of hip girth by $7 \%$; a decrease of crotch girth by $16 \%$ and an increase of center leg length by $10 \%$.

Despite that, the comfort perceived by the user can be a result of a variety of factors, most of them related to the garment or to the wearer him/herself (e.g. satisfaction), which can be both intrinsic, such as the physical characteristics and materials, and extrinsic, such as the brand labels, price etc. (Schutz et al. 2005).

Ideally clothing should not be too loose or too tight; it should provide sufficient ease and enough elasticity to allow workers to move comfortably and in an uninhibited manner (Dorman \& Havenith 2007). If the garment is inappropriately fitted it can adversely affect the wearer's mobility and, for protective clothing, can impact the level of protection (Huck et al. 1997). Park et al. (2015) gave some examples of these effects as:

- The crotch length that when too long, may prevent a worker from moving quickly or may tear and leave him/her vulnerable to hazardous materials;

- The case of a worker who finds his/her clothing uncomfortable may be tempted not to wear the protective clothing at all.

In fact, the Occupational Safety and Health Administration (OSHA 1992) has already issued safety guidelines and regulations to address the subject of loose clothing worn by workers. Extremely loose garments may compromise movement, requiring added movement or force to complete the desired tasks, thereby increasing the metabolic cost of work (Nunneley 1989). Furthermore, performance degradation can also be measured as decreased range of movement, impaired dexterity, reduced speed and decreased accuracy (Rintamaki 2005).

In the case of tight clothing there is not much information available on its effects and consequently, no standards or directives to control the tightness of clothing. However, some recent studies showed that the use of tight trousers causes the restriction of multiple joints in the lumbo-pelvic and hip regions, leading to alterations in the trunk's muscle activity (Anders et al. 2005) and in the person's motion patterns and biomechanics of the remaining unrestricted joints (Yoo \& Yoo 2012). All of these factors can contribute to increasing complaints for low-back pain and disability (Eungpinichpong et al. 2013), neurological problems, heartburn, reflux, as well as bladder problems (Gupta et al. 2014).

For the users it is fundamental that their requirements are met, regarding both appearance and comfort. Currently, with the evolution of the apparel industry to a mass customization paradigm, there are new production techniques that have the potential to adjust clothing to individual preferences (Cho et al. 2005). Standard mass produced ready-to-wear garments have multiple fit issues. According to Hashizume et al. (1999), surveys show that there are both demands and complaints to the current sizing system, for example the existence of few sizes and types for obese and slim customers, since most designs cater for average body sizes, or the difficulty to find suitable pants due to the fixed ratio between hip, thigh and waist sizes. Many of the sizing systems currently in use are reported to be obsolete and useless, as they do not provide accurate information on the body measurements (Faust et al. 2006). These systems use, most of the time, body measurements that are not regularly updated to reflect current anthropometric data, or that represent only a targeted group of consumers because the ready-to-wear industry is still not able to offer tailor-made garments for the most price-sensitive market segments (Faust \& Carrier 2010).

To avoid these issues, customers can opt for made-to-measure bespoke tailoring, where the several items of clothing are designed to meet their specific requirements and body measurements. Despite the fact that this alternative is nowadays the most exquisite and expensive way of buying clothing, this was the only form of clothing production available before industrialization. Nevertheless, this 
approach is more common for specialized clothing products such as space suits for astronauts and performance wear for sports champions (Gupta et al. 2014). This new hyper personalized way of producing clothing is becoming a high-tech revolution, especially with the use of 3D body scanning systems and 3D printers (Daanen \& Hong 2008). The mass-customization approach is becoming more and more accessible to the general public as there are now several systems available that can make single pieces of clothing according to precise individual specifications using advanced CAD and CAM tools (Cordier et al. 2003). The easy access to this type of technology will allow this approach to become more and more popular in high-end fashion, sports-wear, work-wear, clothing with wearable technology and clothing for disabled people. Work clothing should not cause any impedance or restrictions, allowing users to perform daily tasks without stress. As such, the design process should be done using modeling tools that allow the evaluation of the impacts that different designs have on the workers performance (Man and Swan, 2007). To achieve this, garments can be mathematically characterized and modeled to permit some interaction with digital human models. Additionally, the same authors propose a clothing modeling framework to quantify the mechanical interactions between a given clothing system design and a specific wearer performing defined physical tasks. Depending on the age, health and strength of users, as well as the type of clothing in use, the task of handling clothing (donning and doffing, zipping and unzipping, etc.) can be rated from simple to quite complex.

There is a growing concern about comfort of the clothes worn at the workplace; however, designers still tend to overlook the engineering issues for the sake of aesthetics. In a study of Hsiao and Halperin (1998) a six-step guideline for anthropometric design is suggested which, according to Gupta et al. (2014) can be adapted to design of clothing in order to maximize the comfort, satisfaction and performance of the users:

- Determination of the critical body dimensions to the design of a particular clothing type;

- Determination of the target population;

- Determination of the percentage of the population to accommodate;

- Collection of necessary data for the target population;

- Analysis of the data (univariate, bivariate, multivariate or 3D shape analysis);

- Selection of the correct anthropometric design approach for the development of a sizing system.

Designing specific parts of the garments with different properties is a common solution to provide wider movements. According to Ashdown (2011), to achieve this there are various possibilities, such as to:

- Introduce more flexible fabrics (e.g. a stretch fabric in a panel under the arm);

- Increase the flexibility of a fabric with design features (by using pleating or gathers);

- Treat a fabric differently in different areas (e.g. a knitted gardening glove with rubberized areas).

The use of flexible fabrics is especially important when the garment design is intended to be form fitting. Only by using this type of fabrics is it possible for users to move more freely. The knit structure in fabrics provides inherent stretch as it is a looped structure created from continuous threads that are free to shift and move under pressure, but that recover their shape when the pressure is released. In fact, according to Denton (1972) the stretch garments can even be categorized in (i) comfort stretch (the garment is not too close to the body, but it stretches with movement); (ii) stretch-to-fit (the garment fits closely to body but does not apply significant pressure); and (iii) power stretch (the garment exerts compressive pressure on the body). 
In Ashdown (2011), the creation of pattern shapes for active positions is also identified as a good design strategy for increased freedom of movements. Moreover, Watkins (1995) indicated three creative design strategies that can be used to increase movement in clothing:

- Overlapping or stacking segments of inflexible materials next to one another;

- Isolating segments from one another and tethering them directly to the moving body part;

- Creating rigid joints that can roll, slide, rotate, or twist.

The materials used have also a great impact on users' comfort. There is a variety of new high-tech materials that are being used for diverse applications, including work-wear. The type of fibers, or the combination of fibers, used to create a specific fabric affects the properties and the cost of the garments (Ravandi \& Valizadeh 2011). Nowadays it is possible to find new and innovative fibers that possess special properties with web forming technologies and improved chemical and mechanical finishes (Gupta 2011). Based on the end user(s) requirements, the fabrics can be composed by a combination of polymers, metals, ceramics or composites. Still, whichever combination, more functional workwear should be lightweight, elastic, antimicrobial, aesthetically pleasing and durable (Chan et al. 2016). In some very specific fields of work, the fabric can have such a treatment that can make them be UV, bullet, impact, fire, abrasion, and cut resistant. Gupta (2011) gives the examples of three high-tech textiles that are already being used in different areas: smart textiles (react to changes in the environment - e.g. change shape with change in temperature), biometric textiles (mimic and enhance the skin function and performance - e.g. breathable wet suits that are based on the pores of leaves), and e-textiles (incorporate electronic components - e.g. conductive yarns and sensors).

The purpose of this paper is to present the process of creating two prototypes of a women's base upper body garment that is adaptable to different postures and users' requirements. The prototypes were developed in partnership with two specialized companies and were based on information collected from people with different work environments.

\section{Materials and methods}

\subsection{Participants}

Fifty participants volunteered to take part in this study. The participants were divided in two groups. Group 1 (G1) was composed by 30 participants, 12 females and 18 males, with ages ranging from 20 to 62 years old. These participants worked in three different companies/institutions - one research center, one software development company and one university. They were grouped together since they had very similar work characteristics and worked for approximately eight hours in a sitting position, with some occasional standing. This group of workers are free to wear clothes of their choosing as long as they followed the appropriate dress code instituted by the institutions/company;

Group 2 (G2) was composed by 20 participants (all males) with ages ranging from 24 to 59 years old. These participants worked in an industrial company. All these participants work in the day shift for eight hours with a standing posture with some movement. This group wears the company's uniform that consists of a cotton t-shirt, a pair of loose pants and a jacket.

A formal contact was established with the companies, inviting them to participate in the study. The participation in this study was voluntary and the management board of the company selected the participants. When contacted, the participants were informed of the detailed procedures and requirements of the test. 
Table 1 shows the characteristics of the sample population studied (Mean \pm Standard Deviation).

** Table 1 should be placed here **

\subsection{Data collection and analysis}

At the beginning of each session all the procedures and the purpose of the study were explained to the participants. They were informed that the study consisted of two distinct parts: one to collect information about the garments they normally use during their work; and other to gather their anthropometric data that would be used to design new garments.

The data for the evaluation of the workers' satisfaction with their work-clothes and the identification of garments' characteristics was done using a questionnaire. Five different issues were taken in consideration: (i) fit; (ii) mobility; (iii) comfort; (iv) protection; and (v) donning/doffing. The results of this questionnaire are outside the scope of this paper and were already presented in Bragança et al. (2016a).

The anthropometric data was collected by a single certified anthropometrist using traditional methods (one measuring tape and one Harpenden anthropometer). The basic body measurements required to design base patterns (GUDI, 2007) were collected, representing a total of 25 body measurements. The results and analysis of the anthropometric data collected were also already presented in Bragança et al. (2016b).

As the differences between what happens when people changes postures were sought, some measurements were collected in different postures. These were the posture where more significant differences was expected (Figure 1)

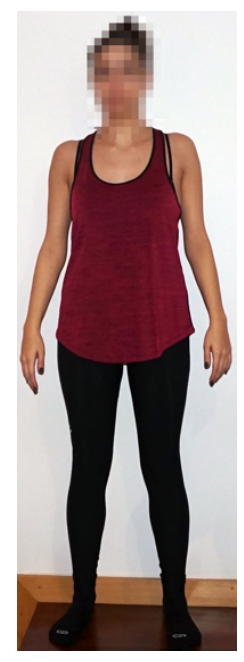

P1

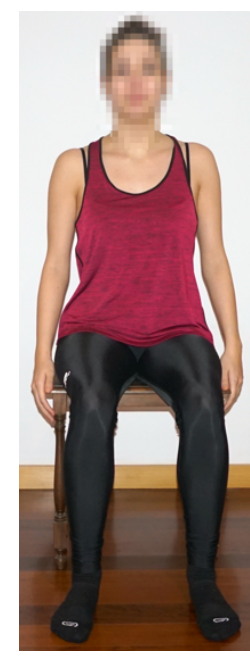

P2

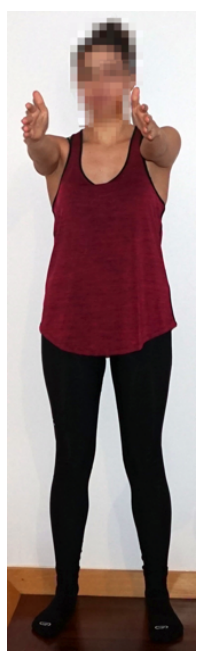

P3

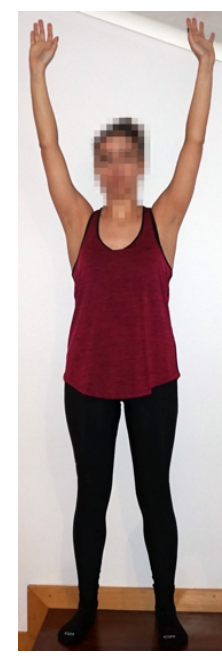

P4

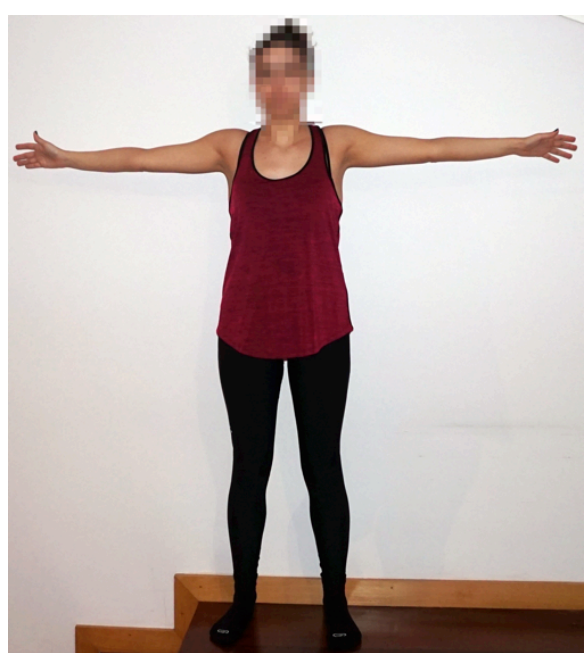

P5

Figure 1. Postures considered for this study.

With all the data collected, the prototypes were developed with the help of two specialized companies: Weadapt - Inclusive Design and Engineering Solutions and FYT Jeans. The first one is responsible for the development of different products designed for people with special needs, such as wheelchair users and the second one holds different patented designs aiming to improve comfort and performance with garments. More recently this company has also developed pants meant for all users, in an everyday or professional use that are adjustable to the several postures adopted by 
people during the day, both in dynamic or sedentary positions. The prototypes presented in this chapter for the upper body follow the same ideas, as the jeans (pants) already developed and communicated by the company and were already submitted for intellectual property protection.

A single participant was selected to use the body measurements needed to create an initial prototype form-fitting base model as well as to wear test different prototypes and perform all the necessary tests. This person was selected due to her availability and because her anthropometric measurements were between the mean and plus or minus one standard deviation of the population analyzed in this study (e.g. Neck base girth $=364.0 \mathrm{~mm}$ that is between the interval $421.4 \mathrm{~mm}(\mu+$ $1 \sigma) 371.0 \mathrm{~mm}(\mu-1 \sigma)$ or Stature $=1655.0 \mathrm{~mm}$ that is between the interval $1777.7 \mathrm{~mm}(\mu+1 \sigma)$ $1639.3 \mathrm{~mm}(\mu-1 \sigma))$. Using the ESMOD methodology (GUDI, 2007) the construction of the prototypes began with the creation of two base patterns for the women's upper body. One using user's personal measurements collected in the static position and the other using the measurements collected in the P3 dynamic position. This process, helped to identify the areas of the garment that needed improvements.

After selecting this person all the necessary anthropometric data was collected. Figure 2 shows the steps followed to obtain the two base models in fabric. The decision to start with a pattern design methodology for a woven fabric, with no stretch, was based in the fact that it would be the most challenging material to achieve the relevant comfort levels. As shown previously the use of stretch materials can reduce the impact of garments during the daily movements of the user.

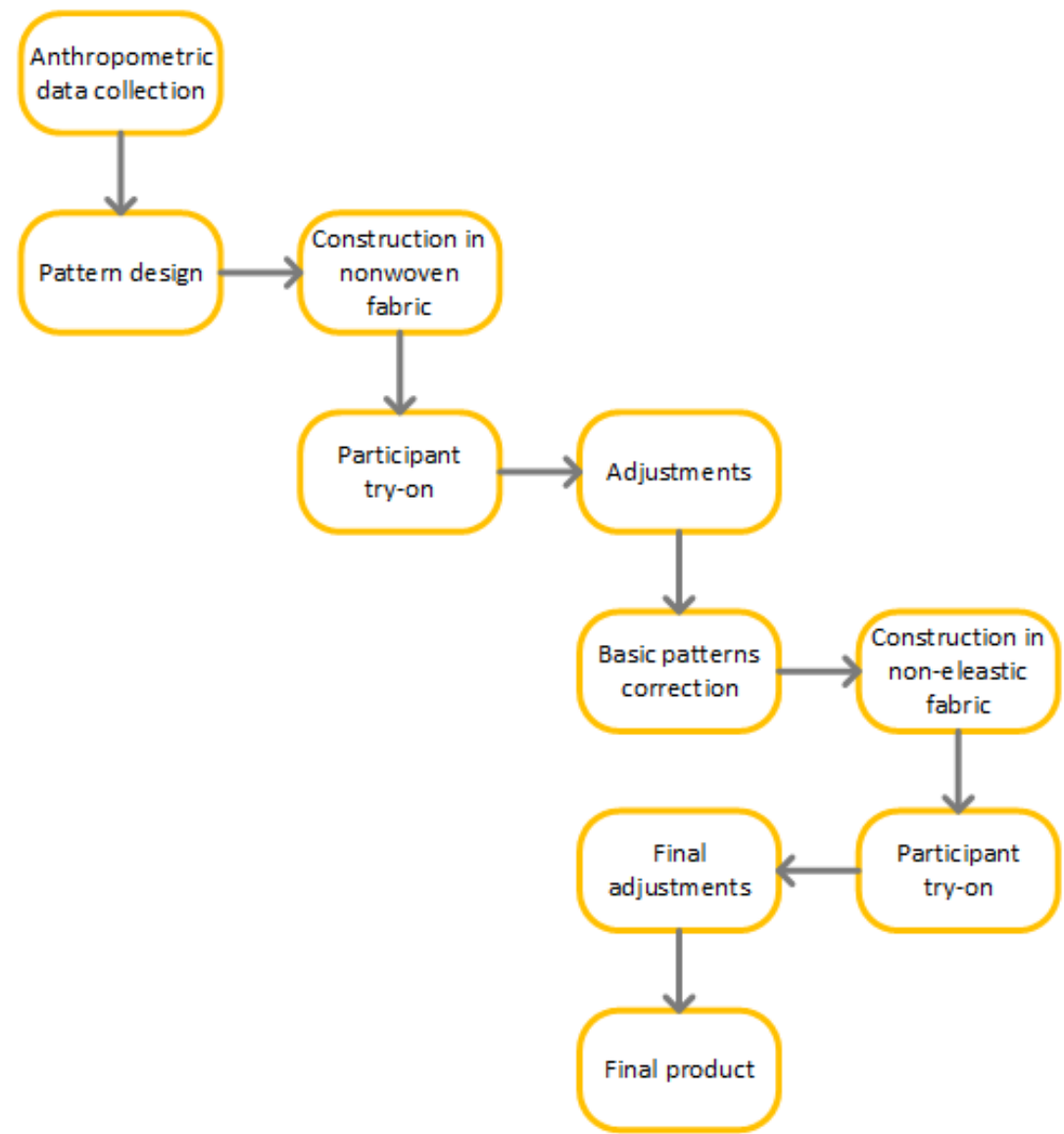

Figure 2. Steps used to obtain the final base models in fabric. 
The first base model was constructed with the measurements of the participant in position P1, whereas the second base model was constructed with the measurements of the participant in position P3. This choice was based on the fact that P3 was the posture where a largest variation was registered (an increase of $14.13 \%$ in the across back length for this particular participant).

A non-elastic woven fabric was used to construct the two base models, fitting the garment as close to the skin as possible. The woven fabric selected is a plain fabric composed of $43 \%$ cotton and $57 \%$ polyester. This characterization was performed using expeditious methods. First of all the fabric was analyzed in the microscope, which reveled the presence of two fibers, one natural and one synthetic. After that, to test the presence of polyester a chemical assay with sulfuric acid was performed. As the fibers were completely dissolved it was possible to conclude that the fabric is composed by cotton and polyester. Lastly, another chemical assay was performed in order to quantify the percentage of each fiber. To do this, the method number seven described in the NP 2248 (1998) standard - mixtures of certain cellulosic fibers or certain elasthanes with polyester: sulfuric acid at $75 \%$ method $(\mathrm{m} / \mathrm{m})$ - was used. Using Equation 1, the percentage of insoluble pure and clean dry compound was calculated.

$$
\mathrm{P}=\frac{100 \mathrm{~m}_{1}}{\mathrm{~m}_{0}}
$$

Equation 1

Where $m_{0}$ is the mass of the pure and dry specimen after pre-treatment and $m_{1}$ is the mass of the dry residue.

This fabric can also be characterized as having 33.8 yarns in the warp and 26.2 yarns in the weft per centimeter. It weighs $116.048 \mathrm{~g} / \mathrm{m} 2$ and has a linear mass of 14.34 Tex for the warp and 12.33 Tex for the weft. The resistance to tear test with a Hounsfield universal tensile machine demonstrated that the warp had a $23.52 \mathrm{~N}$ force of resistance and the weft $26.27 \mathrm{~N}$. Figure 3 shows the results of the five repetitions performed to determine this resistance to tear for both warp and weft.

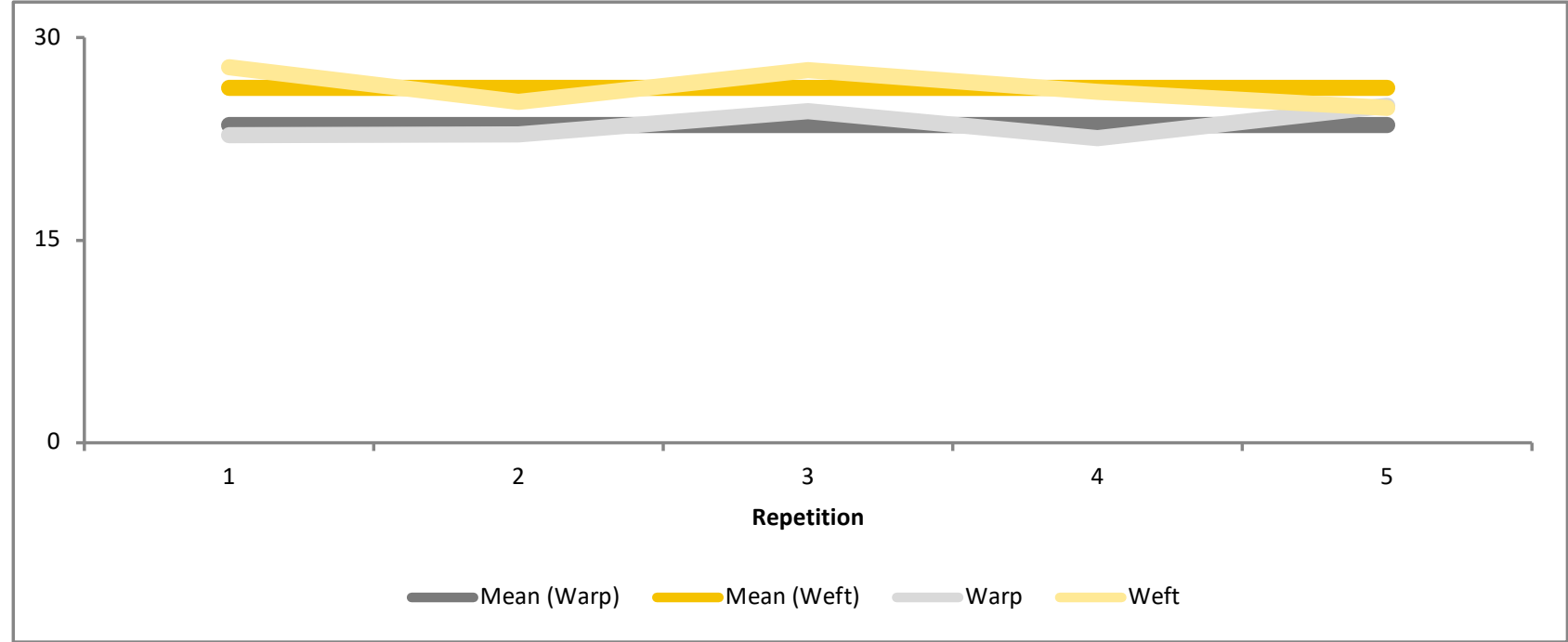

Figure 3. Resistance to tear test for warp and weft.

After the completion of the base models, two different tests were performed. In Test 1, a replica of the first base model (with measurement in P1) was constructed in a nonwoven fabric with low tear 
strength - Figure 4. The participant put on this model and then quickly switched from P1 to P3, with the result that the nonwoven fabric tore at areas of greatest stress. The purpose of this test was to verify which areas of the garment are causing the limitation of movements and which areas are subject to higher compression forces and that consequently tear the low strength fabric as these are the areas where a design feature is needed to provide free movement without binding. In this test the areas where tears were found were meticulously identified and analyzed.
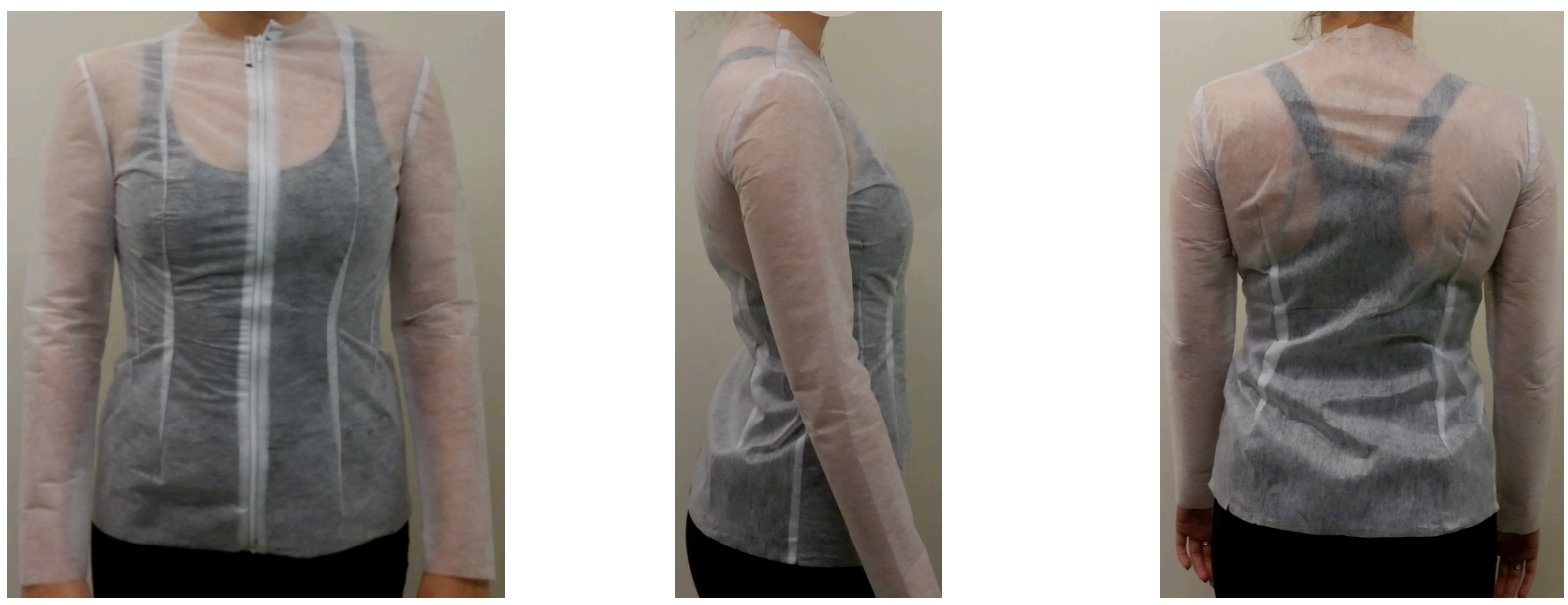

Figure 4. Participant wearing the nonwoven base model for Test 1.

In second test - Test 2 - five compression sensors were used. They were attached to the two base models (constructed with the same woven plain fabric) and were physically connected to a device, which then transmitted recorded data via Bluetooth to a tablet, as can be seen in Figure 5 . The sensors were placed on the locations in which fabric tore when the participant moved from P1 to P3 in Test 1.

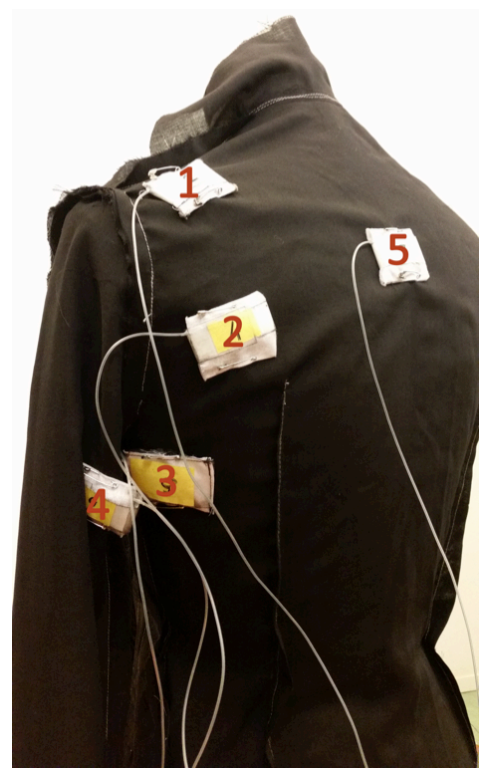

Figure 5. Compression sensors attached to the base model in fabric and their location on the body. 
A new posture (P3v) was added in this test. This new posture was an amplification of the P3 posture, as the participant's arms were crossed in the front, increasing even more the compression forces in some areas. The several postures considered for this test are represented in Figure 6.

Despite the fact that most working task requirements require that people only lift their arms to the front and not cross them, the addition of this new posture was also essential to understand the impact of the exaggeration of the forces to the absolute maximum.
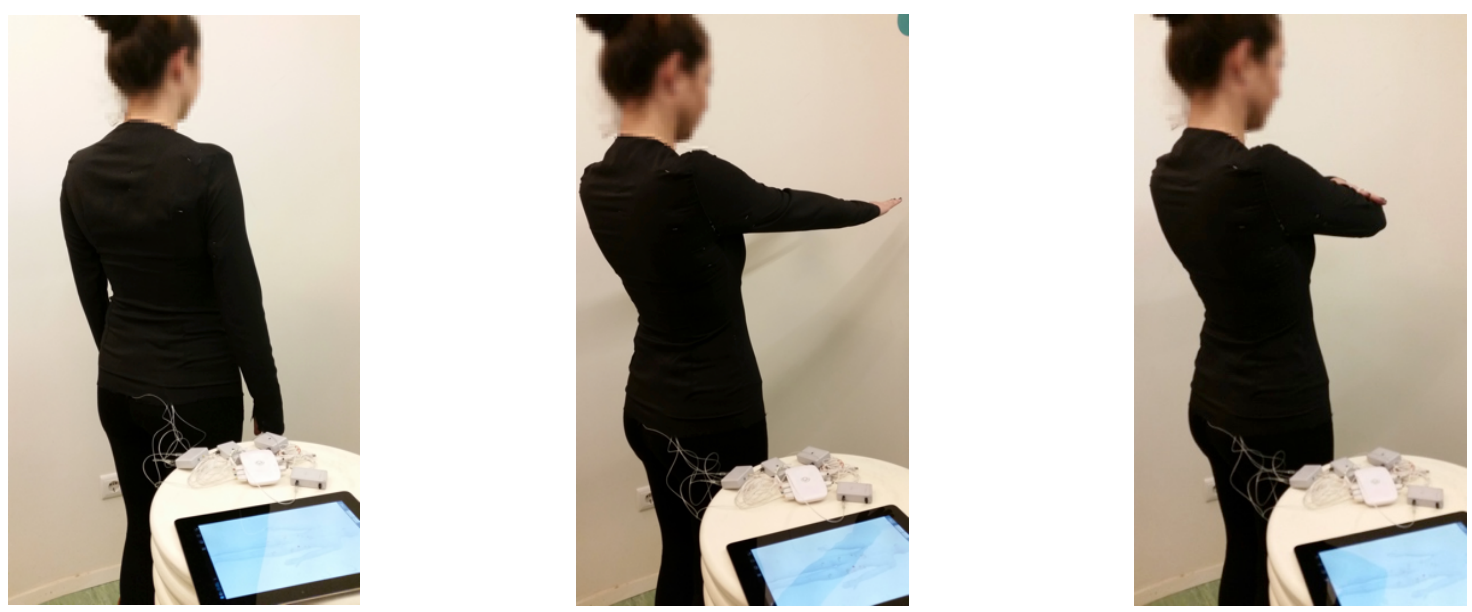

Figure 6. Participant in the several postures for Test 2 (P1, P3, P3v from left to right).

The participant put on the first base model with sensors placed. A first record of the compression values was made with the participant in P1; then a second record took place after the participant switched to P3; and a final recording was made when the participant switched to P3v. This process was repeated three times, making a total of nine records. After this the participant put on the second base model with the sensors identically placed and assumed the three positions again to collect data for comparison. This process was also repeated three times, making a total of nine more records.

This test served the purpose of comparing the compression forces that are present in garments designed considering only the static measurements (static base model) and those in garments designed considering measurements in a dynamic position (dynamic base model). With this test it was also intended to quantify the percentage increase of compression forces when people switch from a static standing posture to a dynamic posture. The mean of the three observations, as well as the variation between postures (P1 and P3 and P1 and P3v) in each base model and between the two base models when in the dynamic posture P3 was calculated. The increase and/or decrease (in percentage) of the compression forces in the various predefined body locations were analyzed.

After the completion of these tests the two prototypes were constructed. These prototypes were based on the static base model. They used the same fabric and had the same dimensions but they were altered with the addition of an elastic mesh. When the prototypes were completed a third test was performed. Test 3 was very similar to Test 2 , both in purpose as in method. The only differences were that (i) the same compression forces were measured for the two prototypes created; and (ii) a new capacitive stretch sensor (equipped with Bluetooth technology and connected to a smartphone - in Figure 7 identified as SS) was also added to record the amplitude achieved in the back and in the armhole areas with the prototypes created.

The main purpose was to quantify the improvement in terms of decrease of compression forces and increase of flexibility that can be obtained with the alterations made to the static base model. It was 
also intended to identify which of the prototypes gives better results when compared with the static base model for the process of switching from a static standing posture to a dynamic posture. In this test the same analysis procedure was applied as in Test 2. Test 3 additionally encompassed an analysis of the stretch ability, with the identification of the percentage variation between the two prototypes and between the postures adopted (P1, P3 and P3v).
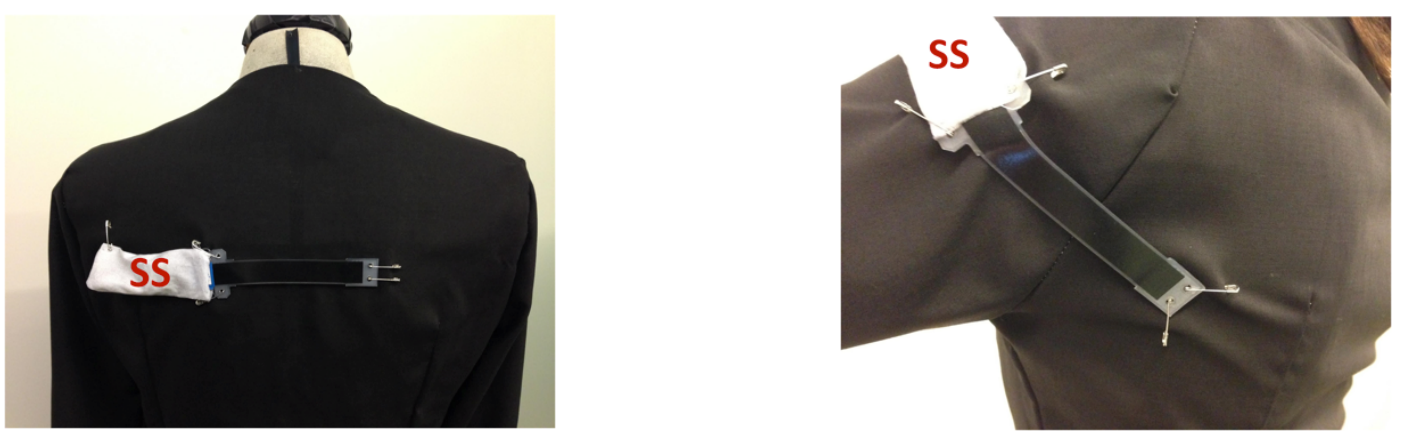

Figure 7. Position of the compression sensors and the stretch sensor for Test 3.

In Figure 8 is showed a summary of all the garments created, their characteristics and the tests performed.
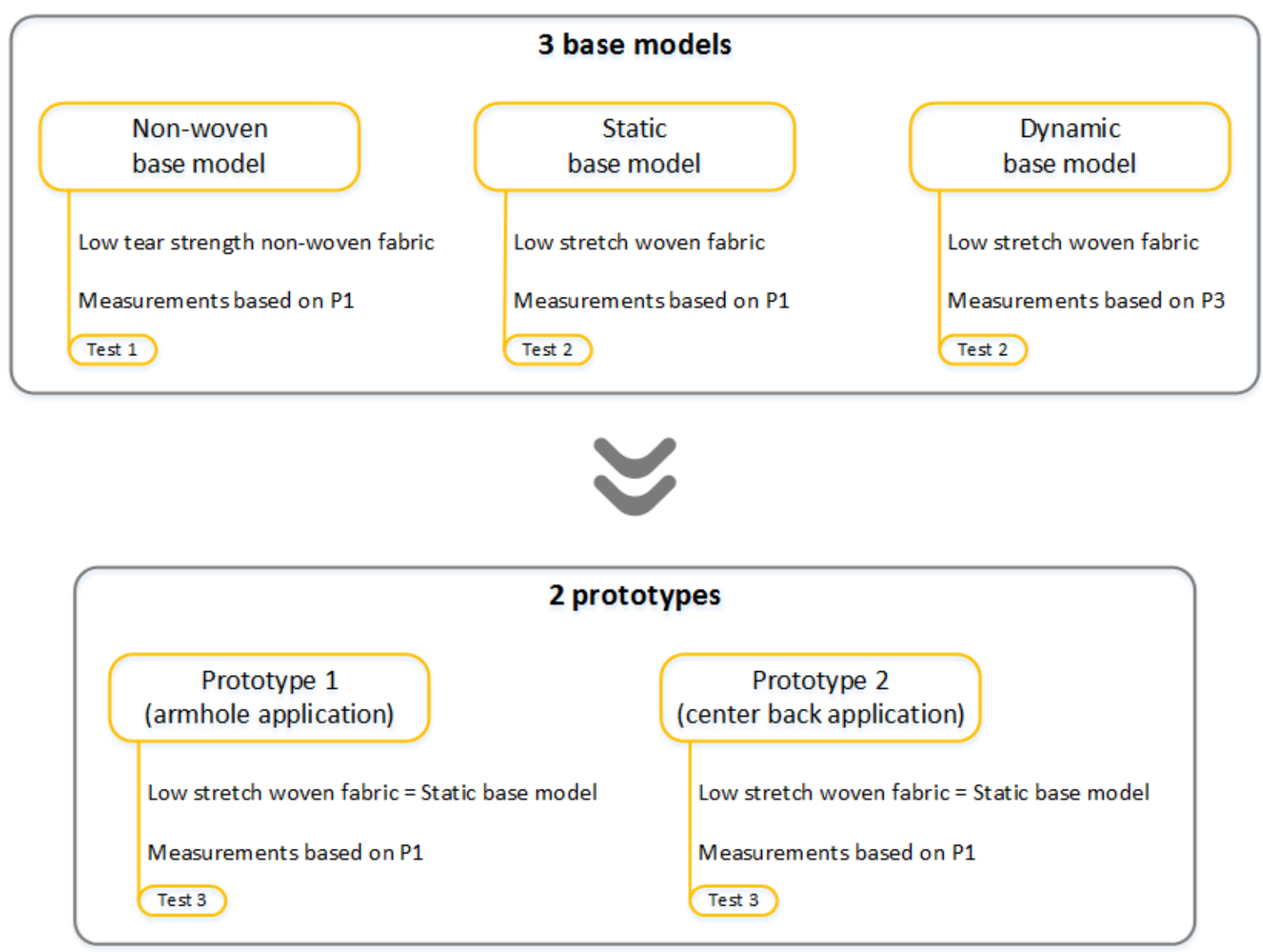

Figure 8. Summary of the garments created. 


\section{Results}

The two base models constructed in the non-elastic woven fabric are presented in Figure 9. As can be seen, both models are skin-tight. However, in the dynamic model there is some slack in the upper back area due to the alteration in the back pattern to accommodate the P3 dimensions (identified in yellow in Figure 10). Nevertheless, to the naked eye and in the opinions of the authors, the dynamic version is not very pleasing in terms of fit and aesthetics, as in a static position there is an excess of material close to the armhole, thus the need to develop more satisfying prototypes.
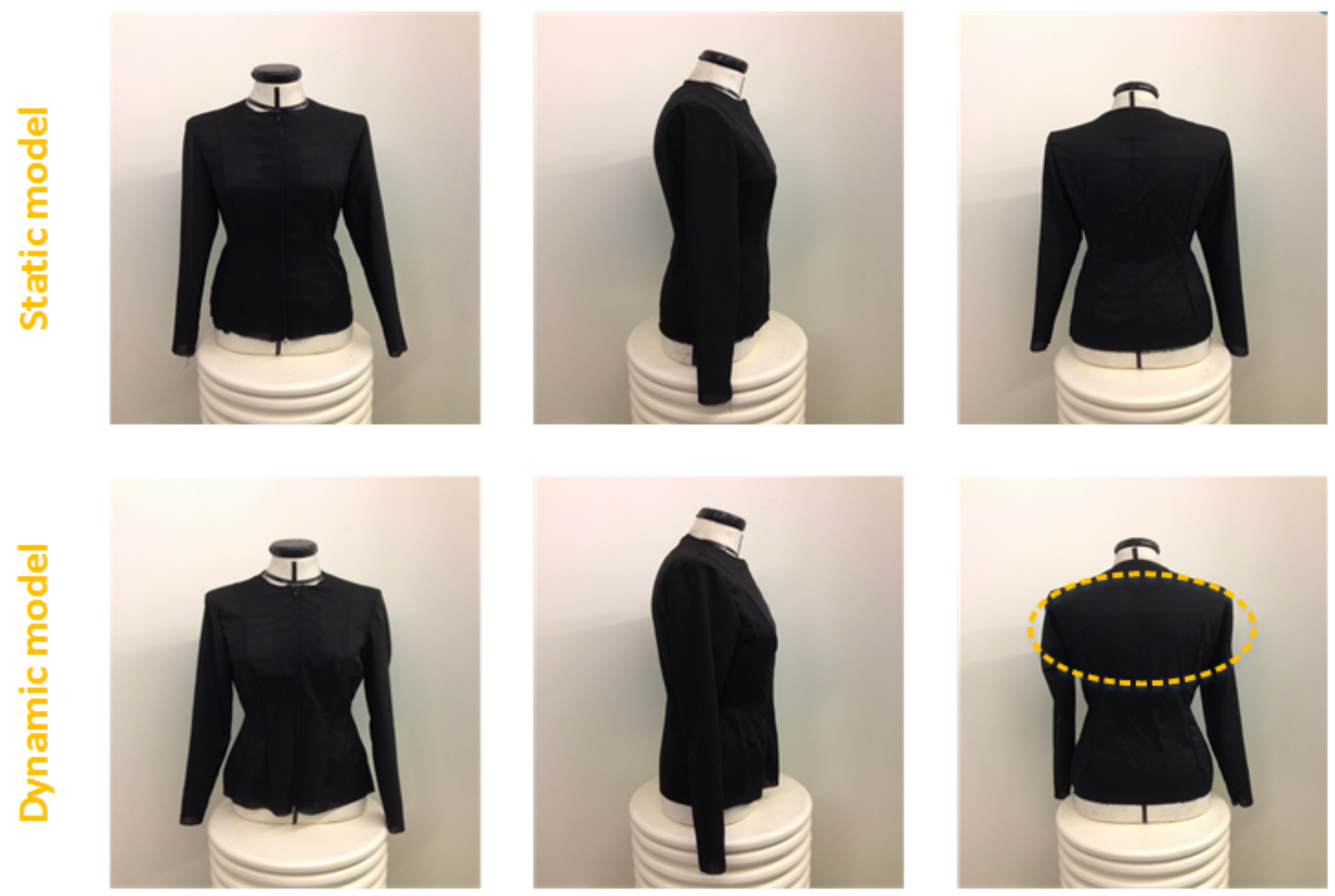

Figure 9. Static and dynamic base models in fabric.

\subsection{Test 1}

The results of Test 1 confirmed that switching from P1 to P3 causes such high compression forces that if the fabric used has low tear strength it will rip. The most sensitive areas are at the joints between the arms and the back, as can be seen in Figure 10. 

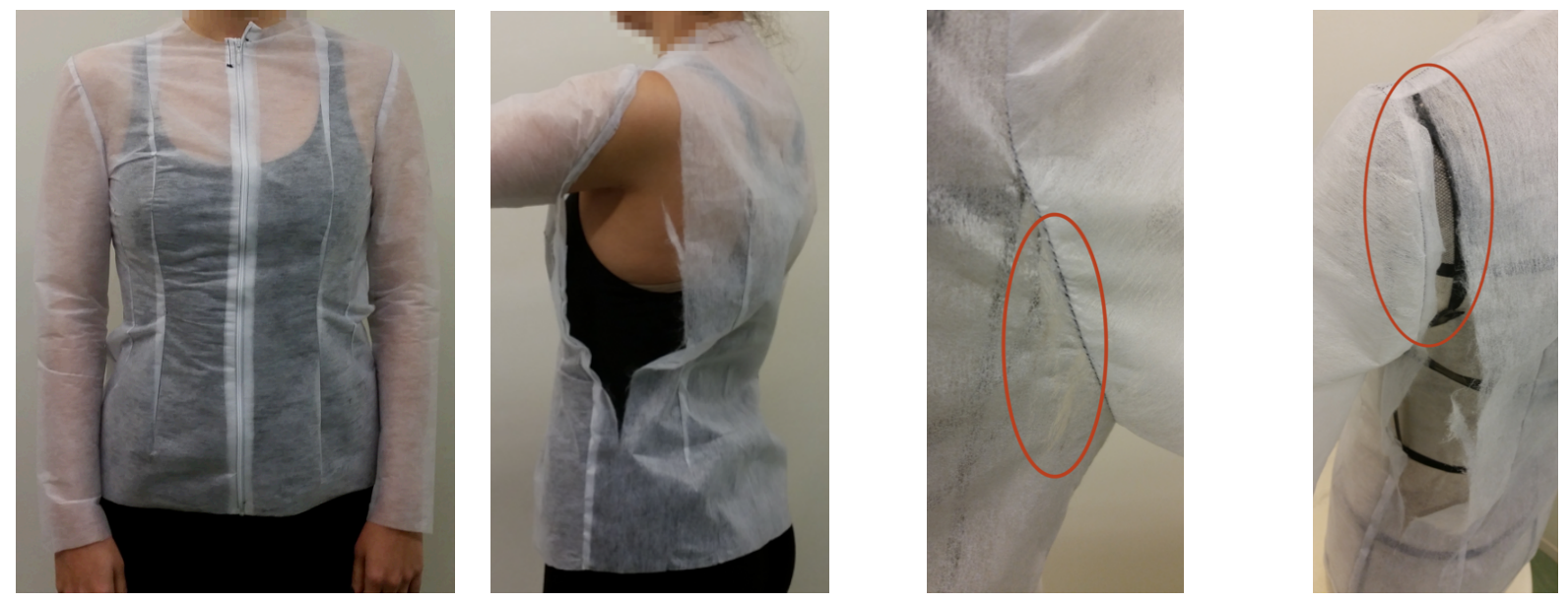

Figure 10. Tears in the nonwoven base model resulting from Test 1.

The front area of the garment remained intact with no visible changes while the back suffered more consequences. In this experiment, only the left side completely opened up. When one side tears, the tightness is released; so major tearing would not be expected on both sides. However, the right side also showed some signs of tearing. As can be seen the rip happened just behind the seam, showing that this did not happen due to seam failures but to fabric failure due to compression forces.

This shows that for the following test the compression sensors should be placed around that particular area.

\subsection{Test 2}

The compression sensors test showed a much higher increase in compression forces when changing from P1 to P3 and from P1 to P3v in the static model. The results of the mean of the three repetitions made in this test for each of the base models can be seen in Figure 11.

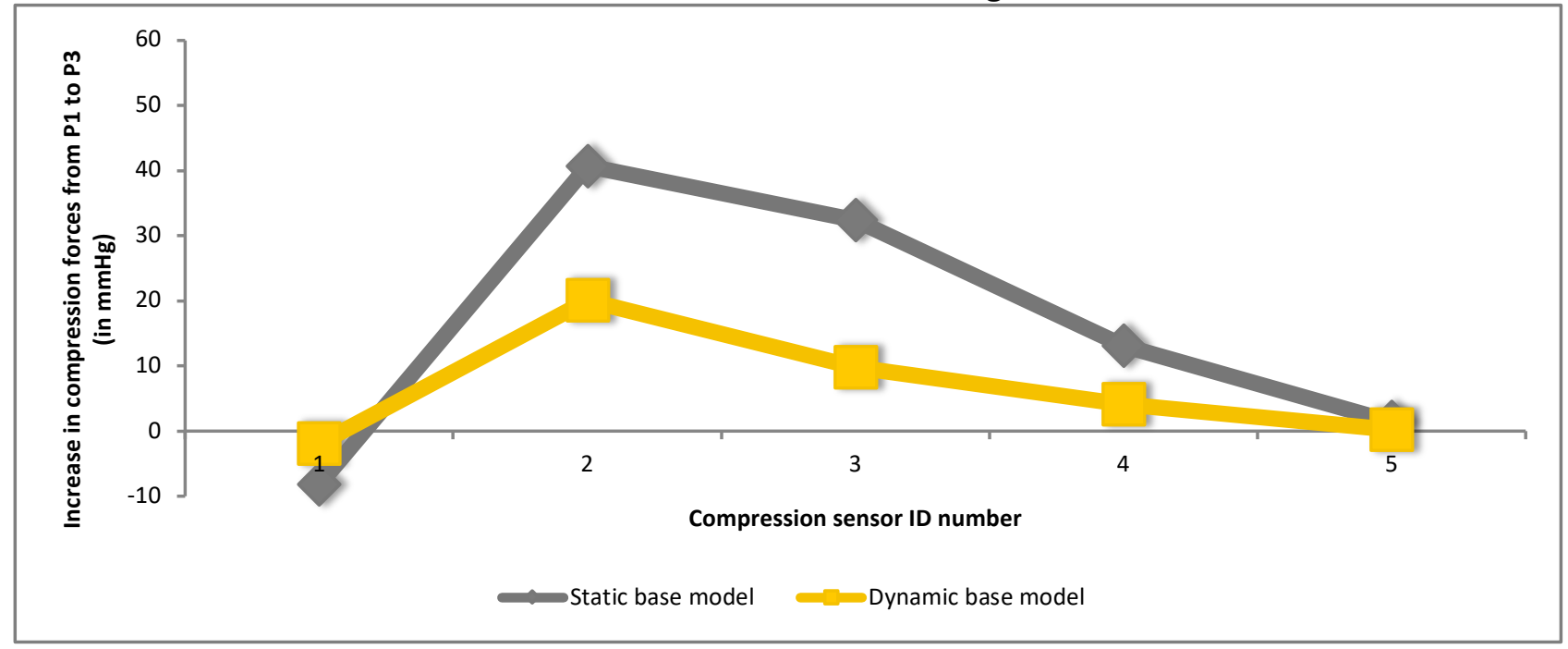




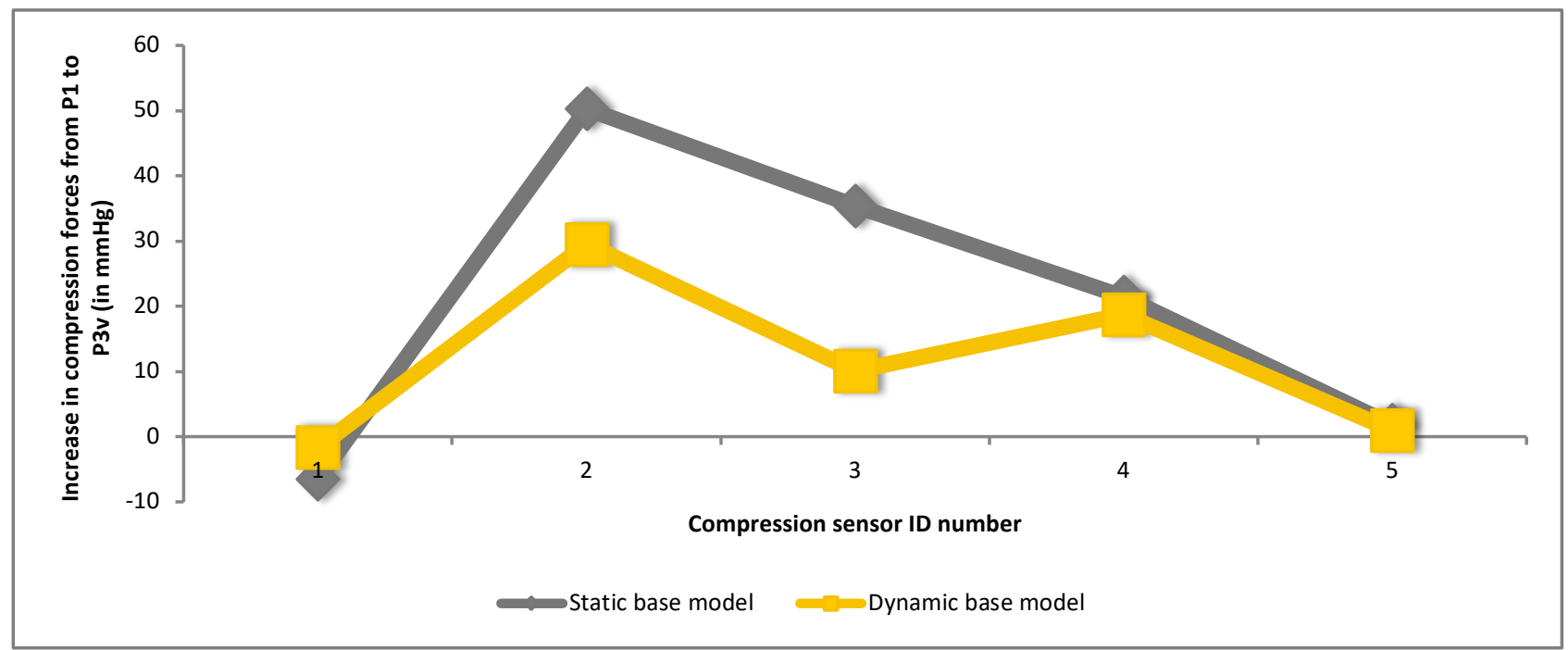

Figure 11. Mean increase of the compression forces when changing from P1 to P3 (up) and from P1 to P3v (down).

It was verified that the compression forces increases very much when the arms are raised, as was expected. There is only one exception, which occurred for the first sensor that decreased its compression value due to the fact that when the arms are to the front the garment becomes looser in the upper area, reducing the contact between the fabric and skin.

The comparison between postures $\mathrm{P} 3$ and $\mathrm{P} 3 \mathrm{v}$ shows that this exaggeration in the posture really has a meaningful impact on the compression forces. All sensors recorded a higher value in P3v than in P3. However, also in P3v the dynamic base model also showed better results.

Comparing the compression forces in P3 and in P3v in the two base models, it can be seen that all the sensors recorded much smaller values when the dynamic base model was used.

The compression forces values for each sensor in P3 and P3v are represented in Erro! A origem da referência não foi encontrada.Table 2. It is possible to see that for both base models and for every sensor the compression forces are higher in P3v than in P3.

The improvements obtained for each sensor when changing from the static base model to the dynamic base model with the participant in P3 are represented in Figure 12. As the results for P3v are very similar they are not necessary to demonstrate.

** Table 2 should be placed here ** 


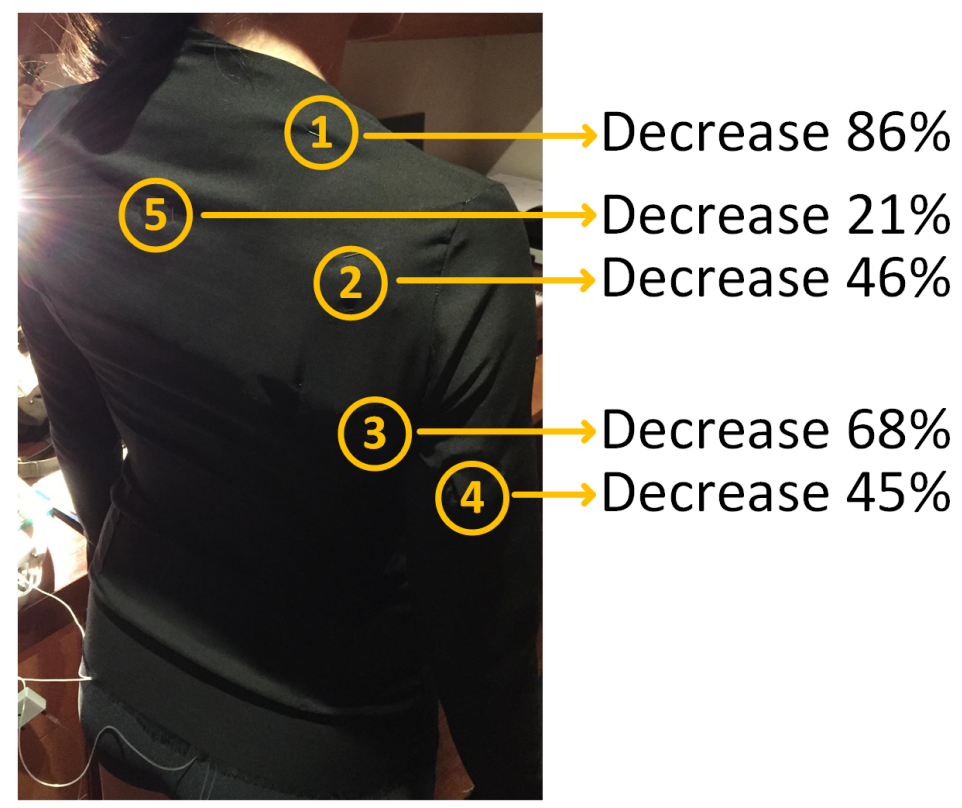

Figure 12. Improvement on the compression forces when in P3 from the static base model to the dynamic base model.

As the static base model was extremely tight the participant had difficulties in raising the arms. Although the participant could move better when wearing the dynamic base model, it was still very form-fitting in the torso and arms. The only difference of this model is that it has more space to accommodate the across back length increase.

Nonetheless, the results obtained show a very significant improvement from the static base model, with the minimum decrease in compression forces of $21 \%$.

\subsection{Prototypes and Test 3}

As previously mentioned, the dynamic base model is not as good as the static one both in terms of general fit and aesthetics. Therefore, to solve the comfort and range of motion limitations problems it was necessary to create a more interesting solution that could be as pleasing as the static model in terms of fit and aesthetics.

Two further prototypes were created, both using the same principle. The main idea was to adapt the base static model, which fits the participant perfectly, using a very elastic mesh (knitting material $100 \%$ polyamide) in some specific areas - the areas that tore up in Test 1 and that were analyzed in terms of compression forces in Test 2. The alterations made allowed the garment to adapt to several postures without compromising the fit and without changing much the general appearance, assuring the aesthetics. In both prototypes the shape and looks are the same as in the static base model. The elastic mesh added only comes into play when dynamic postures are adopted, expanding as needed (in the required direction - up or sides - and in the required amount of space - high or low expansions). It should be noted that, in the examples of the prototypes illustrated here, the mesh used was red so that it contrasted better with the black fabric and was easier to observe and explain.

The first prototype (identified hereon as Prototype 1) had the alterations made to the areas where, according to Test 2, the compression forces were the highest, i.e., the armhole area. A piece of the highly elastic mesh was sewed into the seams of the inner part of the garment. In the area in contact 
with the back part of the garment the mesh follows the delineation of the armhole area (between the back panel and the head of the sleeve) as defined in the pattern design. In the armpit this mesh had a specific flower-like shape to help the mesh to expand and increase the flexibility in several directions. Figure13 shows the placement of the mesh for this first prototype.
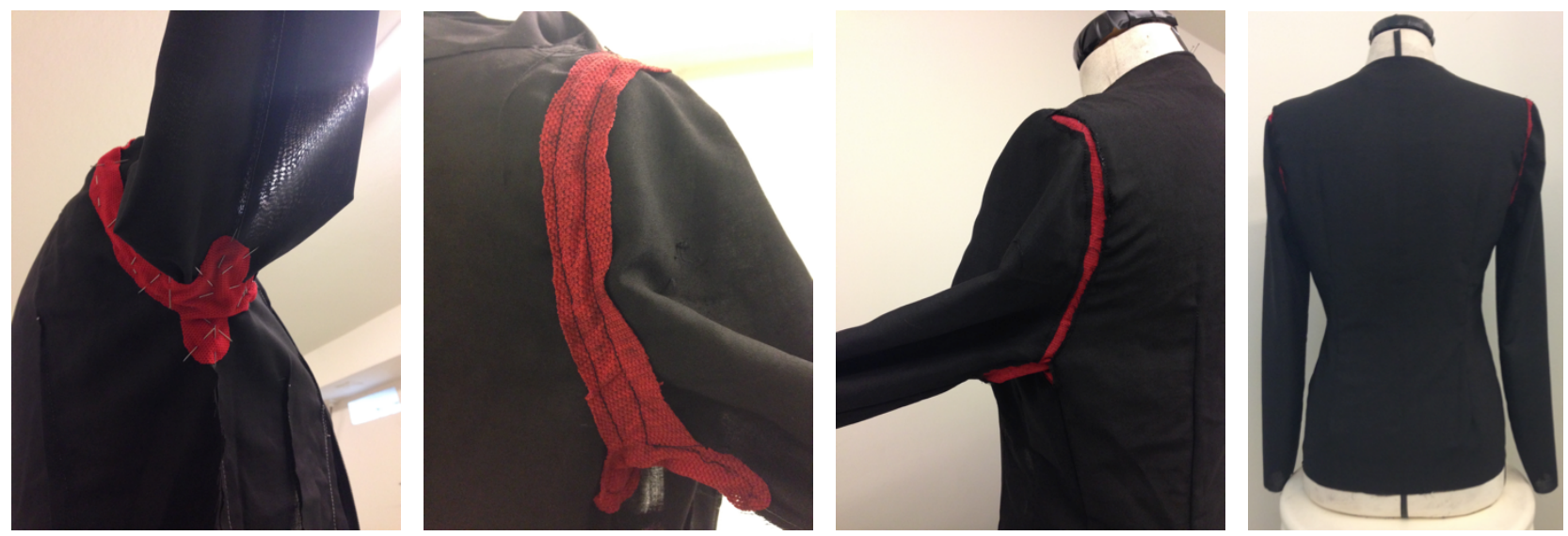

Figure 13. Mesh placement of the first prototype.

In the second prototype (identified hereon as Prototype 2) the alterations to the static base model were made in the back panel, in the center back. In this prototype the fabric of the back was divided in the center back and at the junction of the two parts a small piece of the same highly elastic fabric was added in the inner side of the garment, so it is not visible in a relaxed position. Figure 14 shows the mesh placement of this second prototype. This is a simpler solution but it also allows increasing the flexibility of the garment without making major changes to its general looks.
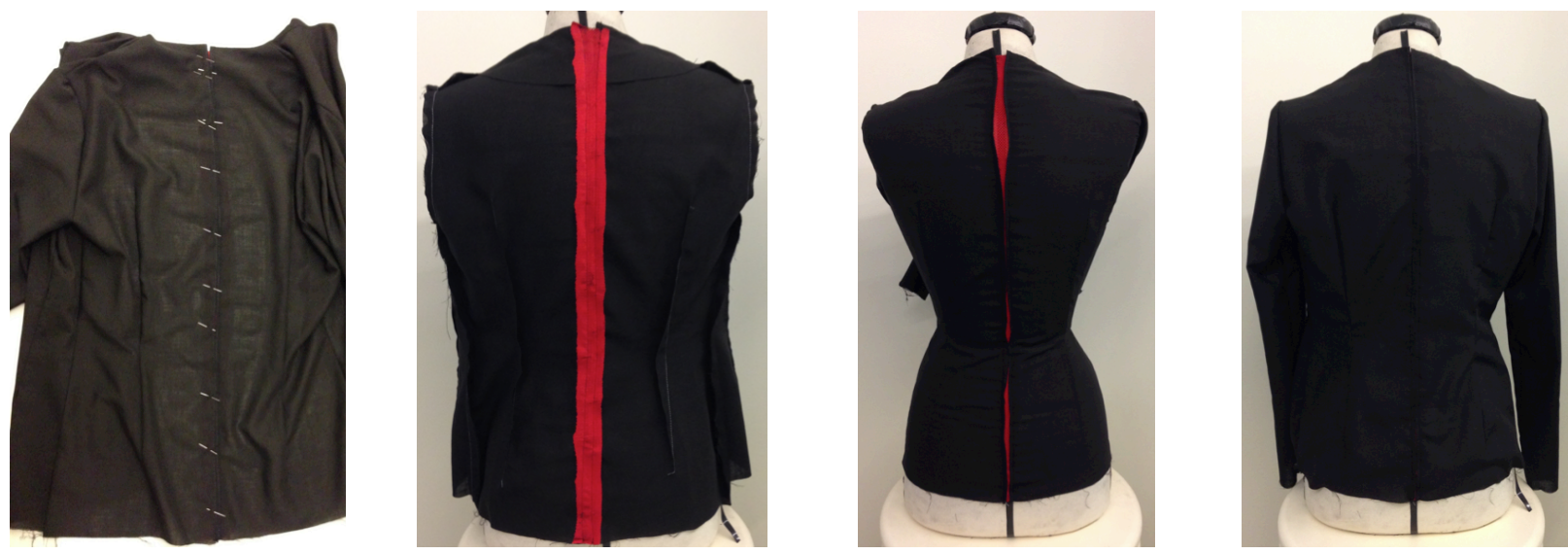

Figure 14. Mesh placement of the second prototype.

The second test with the compression sensors showed a large decrease in the compression forces when changing from P1 to P3 and from P1 to P3v in both Prototype 1 and Prototype 2, with compression forces lower than for the static base model. The mean of the three repetitions made are presented Figure. 


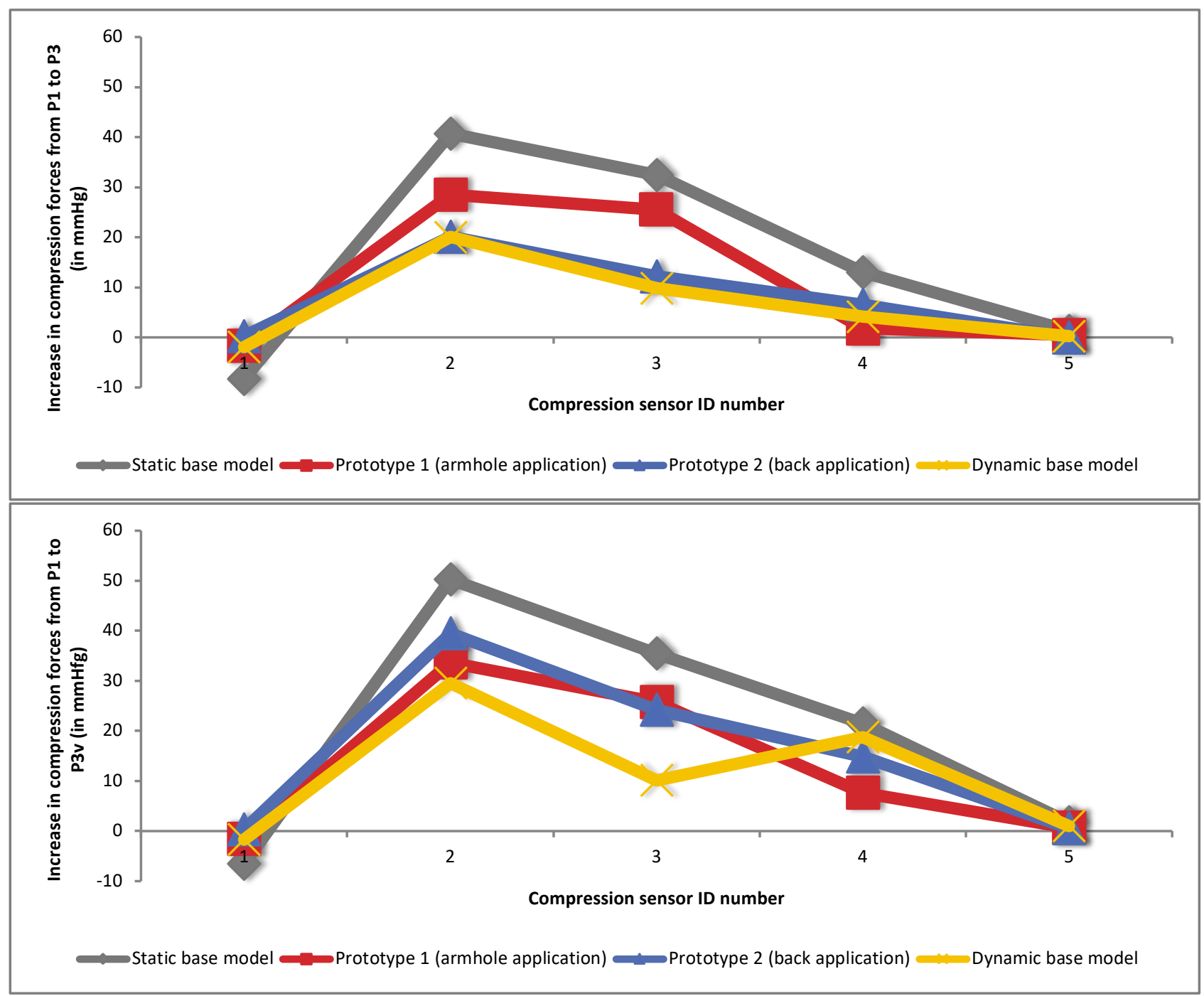

Figure 15. Mean increase of the compression forces when changing from P1 to P3 (up) and from P1 to P3v (down) for the two base models and for the two prototypes.

Interestingly, the Prototype 2 (with the back application) recorded lower compression forces than the Prototype 1 (with the armhole application) in almost all sensors, with the exception of sensor 4 (placed in the sleeve).

In contrast, when the posture is accentuated to P3v, the Prototype 1 shows better results. This is due to the increased flexibility of the Prototype 1 because of the application in the armpit, which allows having more expansion when the arms are crossed in the front. For sensor 1, the Prototype 2 recorded a similar value than the static model, since the back application is not enough to cover for the alteration in that upper back area. Sensor 3 demonstrates very similar results for Prototype 1 and for the Prototype 2, however, the Prototype 1 is better, due to the armhole application. Nevertheless, the dynamic model still had the lowest increase in compression forces for all sensors, except sensor 4 .

When in the most extreme posture, P3v, compared to the static base model, the compression forces decrease drastically when wearing both Prototype 1 and Prototype 2 - Figure 16 . Neither of the prototypes presented the best result for all sensors. The Prototype 1 was only better than the Prototype 2 in sensors 2 and 4. 


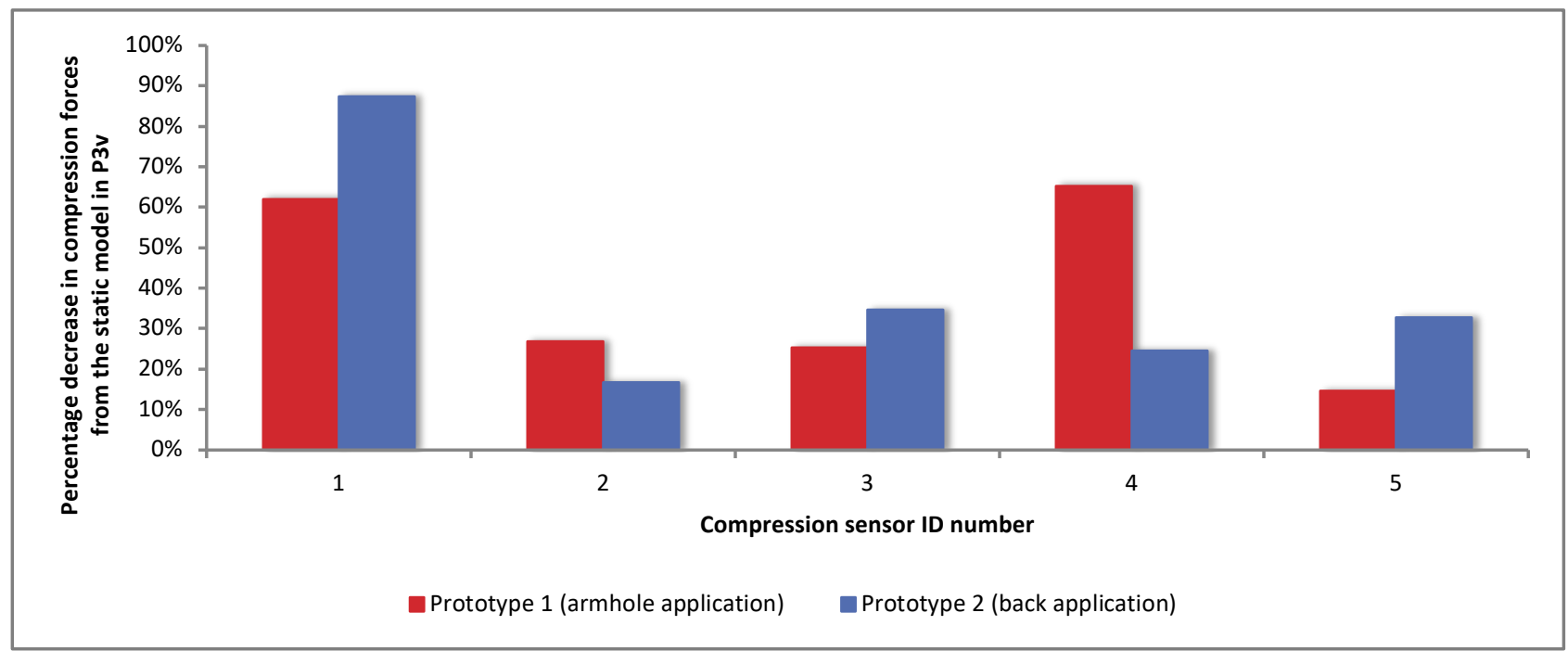

Figure 16. Percentage decrease in compression forces from the static model to the prototypes in P3v.

This shows that there were very impactful improvements if changes were made to the static base model. It is possible to achieve up to $87 \%$ less compression. The lowest decrease registered was $15 \%$ in the middle of the back, which is an area less subject to the compression forces.

In terms of the stretch ability that can be achieved with the prototypes, there is a difference if the back or the armhole area is considered. As can be seen in Figure 17, Prototype 2 is the one that presents the highest stretch ability for both areas and for both postural changes. In the back area the dynamic base model also registered a good stretch ability for both postural changes. In the armhole area Prototype 1 and Prototype 2 recorded good results. 


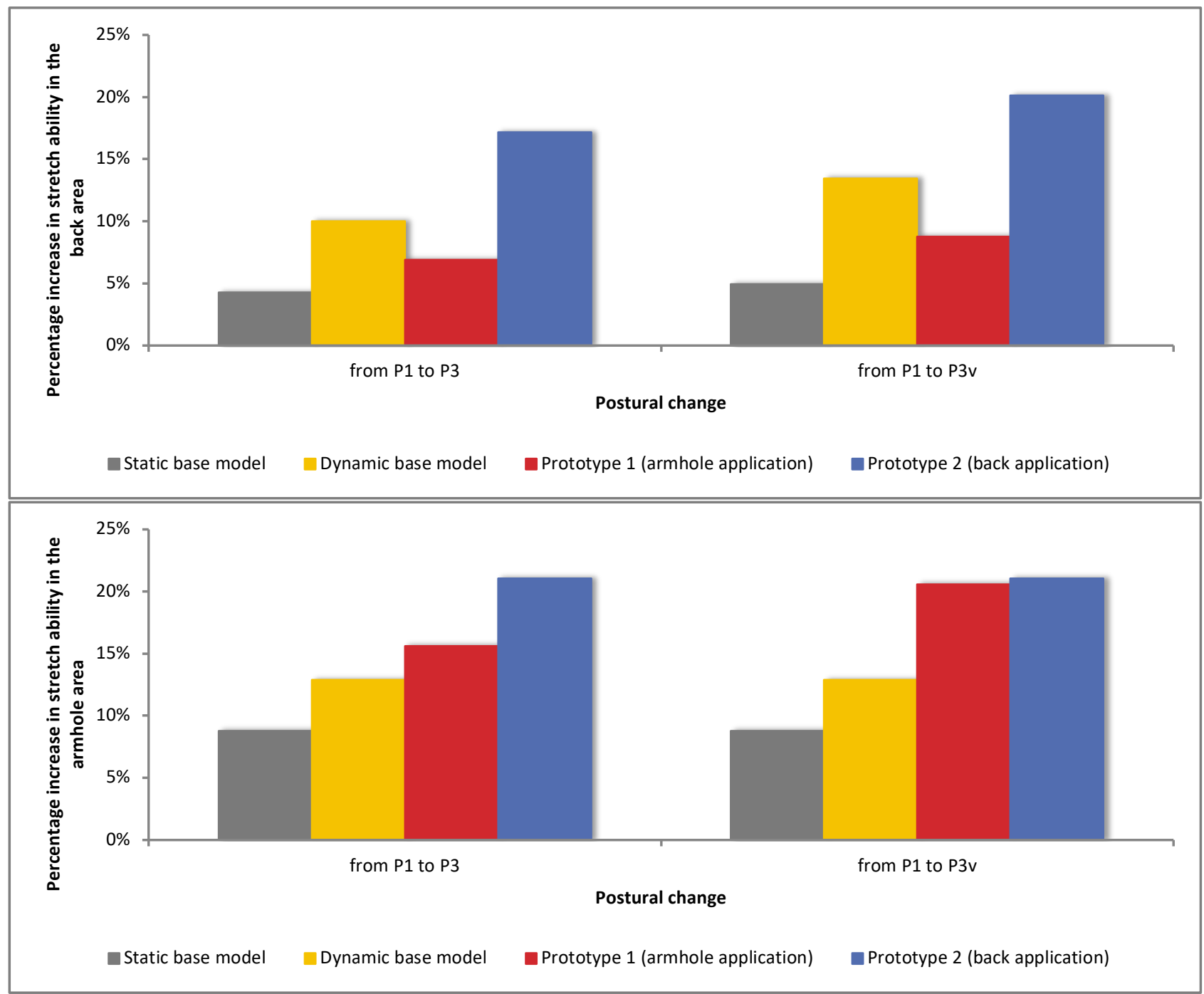

Figure 17. Percentage increase in the stretch ability when changing postures in the back (up) and in the armhole area (down).

In the back area the dynamic base model behaves better than Prototype 1 because of the excessive fabric. Despite the fact that Prototype 1 can be adjustable, its capacity to increase is lower than the excessive fabric in the back area of the dynamic base model. Nevertheless, when the armhole area is considered the Prototype 1 is better than the dynamic base model, as this is the specific area where this prototype is more efficient.

It can also be noticed that when the posture was exaggerated (from P1 to P3v) only the dynamic base model and the Prototype 2 were able to stretch a little more. On the other hand, in the armhole area, the Prototype 1 recorded a high increase in the stretch ability, whilst the other garments remained practically the same.

As can be seen, each prototype revealed better results in the specific area where they had the application of the elastic mesh. As expected, when the exaggerated P3v posture is adopted, Prototype 1 is more flexible in the armhole area and Prototype 2 is more flexible in the back area. 


\section{Discussion}

Most of the clothing is designed for mass consumption, making it possible accommodate a large variety of people in the same size. However, this leads many times to fit problems because people with distinct body types and sizes are intended to wear the exact same size. As such, when designing clothes, features such as good fit, good shaping and high flexibility, should be thoroughly considered.

The questionnaire performed prior to this study showed that the first participant group feels, in general, relatively dissatisfied with their garments. The results of the questionnaire on worker's issues with clothing are in agreement with the anthropometric measurements. The parts of the body with the most variation in active postures in the study are the same as the participants pointed-out as areas of limitation of movements in the questionnaire (sleeves; armholes; shoulders; chest and back; waist; hip; thighs; legs). For a more detailed analysis of the questionnaire findings please see the previous publication - Bragança et al. (2016a).

All the body parts measured in this study, except the leg length, had statically significant differences when the posture changes, proving the great impact they have on garments' fit. The impact and significance of these differences were demonstrated with the tear test (Test 1 ) and the measurement of compression forces (Test 2). This made it clear that if garments are not designed with consideration to the dynamic postures they will affect the compression imposed to the user, and can also limit movements and cause discomfort.

The prototype presented in the first subsection of this paper was designed to accommodate the most significant change that occurs in the upper part of the body affected by the garment's design - the increase of the across back length. The results of the compression forces test (identified as Test 3) between the two prototypes already showed a high decrease in the compression forces exerted when the arms are raised to P3 and to P3v.

As can be seen in Figure 1818 the mesh selected to develop these prototypes has the ability to almost double in size if needed.
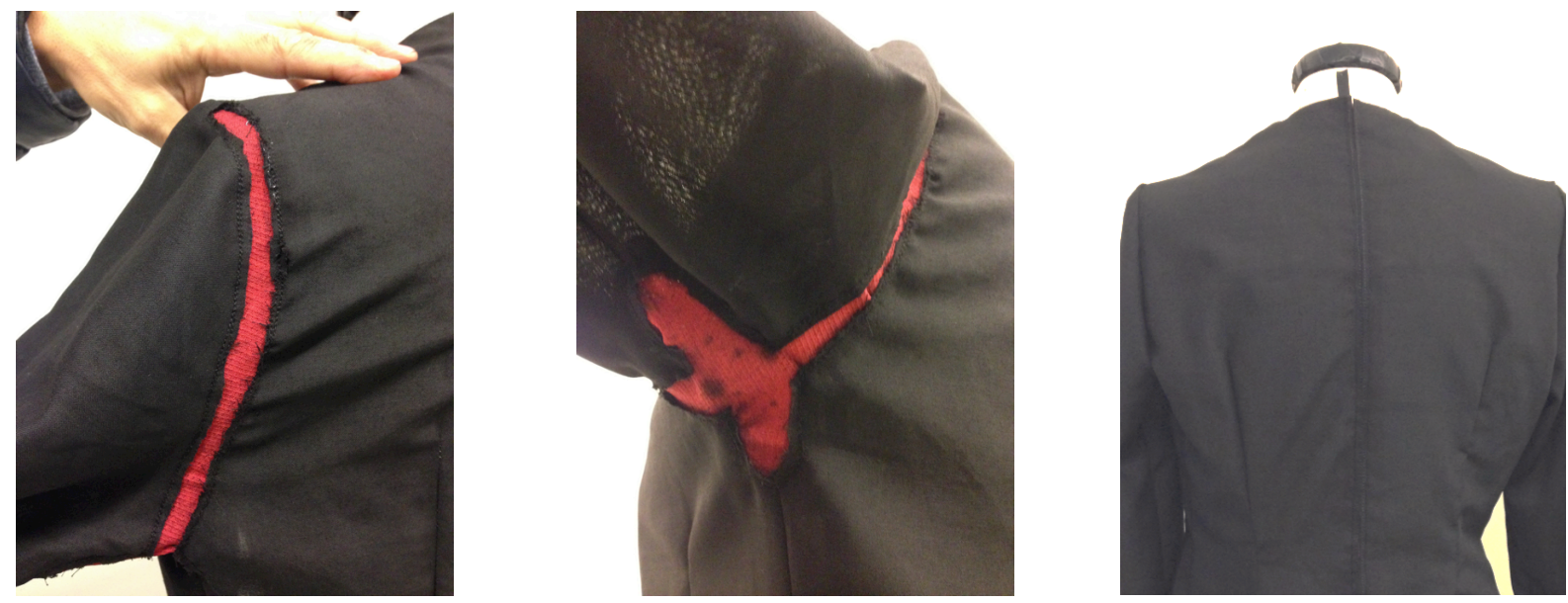

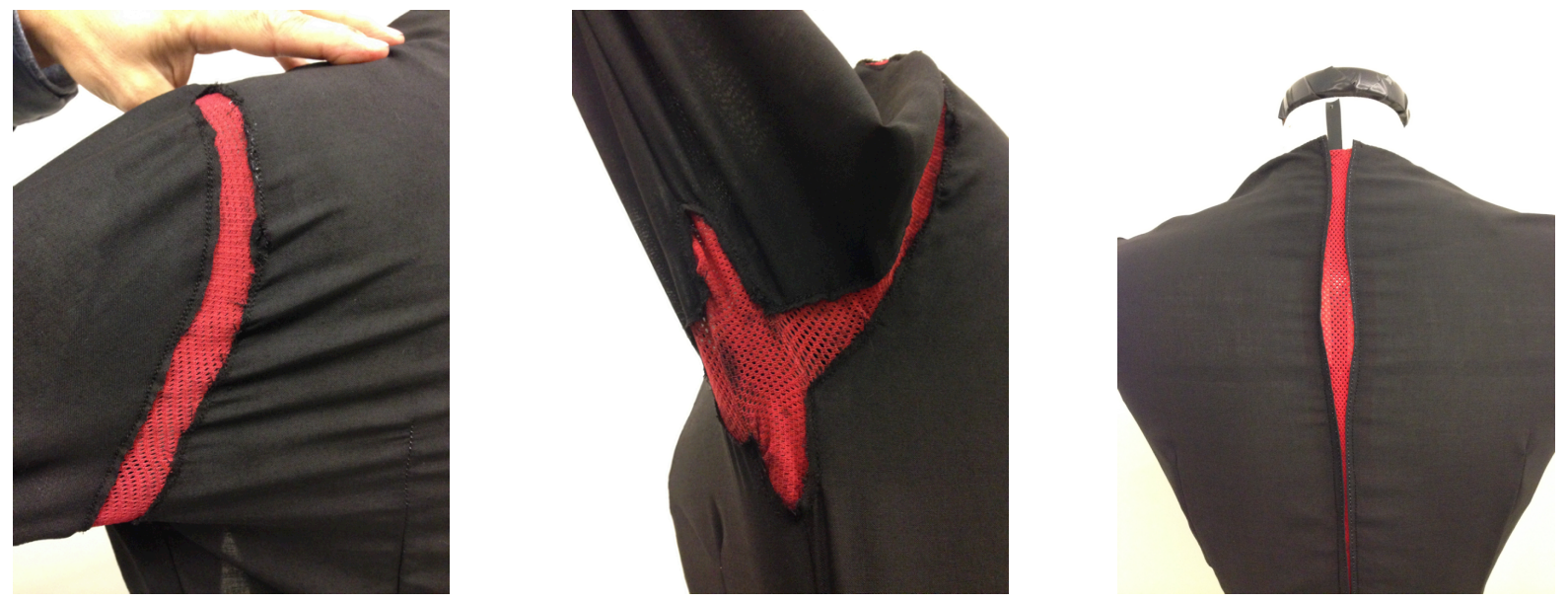

Figure 18. Flexibility of the elastic mesh used.

Taking this in consideration, the exaggeration of posture P3 was very useful to fully understand the impact of the two prototypes. It showed that for less extreme postures, such as P3, the back application of Prototype 2 is enough to reduce the compression forces in almost all the critical parts of the garment. On the other hand, when more extreme postures are adopted, Prototype 2 fails to provide the necessary increase of flexibility, while the application in the armpit of Prototype 1 gives even more flexibility to the garment.

Test 3 revealed that both prototypes behave well when changing from a static posture to a dynamic posture in terms of compression as well as of stretch ability. Neither of the prototypes provided, results for all compression sensors; some showed better results for one prototype and others for the other prototype. This test also demonstrated that the first prototype, the dynamic base model recorded better results than the static base model and the two prototypes. Nevertheless, as previously stated, this base model is not very satisfying in terms of fit and aesthetics. As such, the better results in the compression forces' test do not make up for those negative issues. It would be possible to achieve the same results as the dynamic base model, if some new alterations to the prototypes were added, such as: (i) combining the two prototypes in one, i.e., having both the armhole and the center back application in the same garment; and (ii) using an even more elastic material in the inserts.

Both alternatives previously mentioned would give the garment an additional ability to expand, reducing compression forces even more. In fact, Test 3 results revealed that combining the two prototypes in one would assure good results for all the sensors that were placed, since in the current situation the best results in each sensor vary from one prototype to the other.

This solution that is apparently very simple can bring great improvements to the users' feeling of comfort. These alterations not only increase the freedom of movements but they also facilitate the donning and doffing processes. That feeling that many users have when wearing a shirt (as if they are trapped inside the clothes and even breathing is difficult) is avoided with these prototypes as the shirt molds itself better to the users' needs. These alterations can also be useful to users with some kind of limited dexterity, whether it is a congenital or short term disability, which for example does not allow them to raise their arms to put on a shirt. The examples presented here show that the shirt provides the required flexibility to allow a better fit as well as function and an easier and more efficient use of the product.

In the prototypes presented, the priority was given to functionality, rather than aesthetics. The elastic mesh is visible and the shirt no longer has that classic look. However, from the fashion design point of view, this can be considered as intentional and as part of the garment's looks. The mesh 
selected could be the same color as the base fabric, but again, this option is also valid for a less conservative and classic look.

One of the difficulties faced was in the construction process, which was very time consuming and difficult given the limited production equipment available. However, as the priority was to have a functional product the aesthetic issues introduced in the construction did not have critical importance. Still, if this would be a product to be produced in large scale, the assembly process would require a further study to improve aesthetics and reduce production costs - it would require having appropriate industrial equipment and skilled construction operators.

Another limitation of this study was the lack of opportunity and funding to test the prototypes created with a larger sample size. This would provide an opportunity to have a better understanding of the impact the prototypes have on the users' comfort and satisfaction. Still, this is one of the main objectives for future work.

\section{Conclusions}

Despite the fact that people are becoming more and more sedentary, there is still the need to perform movements and adopt active postures during leisure and work activities that may be negatively influenced by clothing. Some examples of those types of postures are the ones presented in this study, which proved to have quite a meaningful impact on the compression forces and consequent comfort of users.

It is important to create functional clothes that can adjust to people's daily activities and needs. However, this is only possible if the variations that occur in the body with the several dynamic postures adopted during the day are considered. The adjustments made to the garments are essential to guarantee freedom of movements and comfort, which are very important for both labor and leisure activities. If these adjustments were associated with a good looking and fashionable design, these garments would satisfy people who value comfort as well as aesthetics. These designs could also contribute to ease of donning and doffing for people who are disabled and have limited function.

This study overall demonstrated two important issues: (i) the opinions and levels of satisfaction of people who work in different work environments and with distinct work characteristics towards the clothes they wear; and (ii) the differences that can be registered in the anthropometric data when postures change. With all this information, which is not usually taken in consideration in the process of garment design, it was possible to construct two prototypes for the upper part of the body, which can adjust to the users' needs, fostering higher levels of comfort and lower levels of movement limitations.

\section{References}

Anders, C. et al., 2005. Trunk muscle co-ordination during gait: Relationship between muscle function and acute low back pain. Pathophysiology, 12(4), pp.243-247.

Ashdown, S.P., 2011. Improving body movement comfort in apparel. In Improving Comfort in Clothing. Cambridge, UK: Woodhead Publishing Series in Textiles, pp. 278-302.

Chan, A.P.C. et al., 2016. Evaluating the physiological and perceptual responses of wearing a newly designed construction work uniform. Textile Research Journal, 86(6), pp.659-673.

Cho, Y. et al., 2005. An interactive body model for individual pattern making. International Journal 
of Clothing Science and Technology, 17(2), pp.91-99.

Choi, S. \& Ashdown, S.P., 2011. 3D body scan analysis of dimensional change in lower body measurements for active body positions. Textile Research Journal, 81(1), pp.81-93.

Cordier, F., Seo, H. \& Magnenat-Thalmann, N., 2003. Made-to-measure technologies for an online clothing store. IEEE Computer Graphics and Applications, 23(1), pp.38-48.

Daanen, H. \& Hong, S.-A., 2008. Made-to-measure pattern development based on 3D whole body scans. International Journal of Clothing Science and Technology, 20(1), pp.15-25.

Denton, M.J., 1972. Fit, stretch and comfort. Textiles, 3(1), pp.12-17.

Dorman, L.E. \& Havenith, G., 2007. Examining the impact of protective clothing on range of movement.

Eungpinichpong, W. et al., 2013. Effects of restrictive clothing on lumbar range of motion and trunk muscle activity in young adult worker manual material handling. Applied Ergonomics, 44(6), pp.1024-1032.

Faust, M. \& Carrier, S., 2010. Women's wear sizing: A new labelling system. Journal of Fashion Marketing and Management, 14(1), pp.88-126.

Faust, M., Carrier, S. \& Baptiste, P., 2006. Variations in Canadian women's ready-to-wear standard sizes. Journal of Fashion Marketing and Management, 10(1), pp.71-83.

Gupta, D., 2011. Design and engineering of functional clothing. Indian Journal of Fibre and Textile Research, 36(4), pp.327-335.

Gupta, D., Gupta, D. \& Zakaria, N., 2014. Anthropometry and the design and production of apparel: An overview. In Anthropometry, Apparel Sizing and Design. United Kingdom: Woodhead Publishing Ltd, pp. 34-66.

Hashizume, S. et al., 1999. Study on the fittability of clothing size. Journal-Japan Research Association for Textile End Uses, 40(1), pp.45-53.

Hsiao, H. \& Halperin, W., 1998. Occupational safety and human factors. In Environmental and Occupational Medicine. Philadelphia: PA: Lippincott- Raven.

Huck, J., Maganga, O. \& Kim, Y., 1997. Protective overalls: Evaluation of garment design and fit. International Journal of Clothing Science and Technology, 9(1), pp.45-61.

Kirk, W. \& Ibrahim, S.M., 1966. Fundamental relationship of fabric extensibility to anthropometric requirements and garment performance. Textile Research Journal, 36(1), pp.37-47.

Man, X. \& Swan, C.C., 2007. A mathematical modeling framework for analysis of functional clothing. Journal of Engineered Fibers and Fabrics, 2(3), pp.10-28.

Nunneley, S.A., 1989. Heat stress in protective clothing: Interactions among physical and physiological factors. Scandinavian Journal of Work, Environment \& Health, 15(1), pp.52-57.

OSHA, 1992. Concepts and techniques of machine safeguarding, Washington, DC.

Park, H. et al., 2015. Impact of firefighter gear on lower body range of motion. International Journal of Clothing Science and Technology, 27(2), pp.315-334.

Ravandi, S.H. \& Valizadeh, M., 2011. Properties of fibers and fabrics that contribute to human comfort. Improving comfort in clothing. Philadelphia: Woodhead Publishing Series in Textile, pp.61-78.

Rintamaki, H., 2005. Protective clothing and performance in cold environments. In 3rd International Conference on Human-Environment System. pp. 12-15.

Schutz, H.G., Cardello, A. V \& Winterhalter, C., 2005. Perceptions of fiber and fabric uses and the factors contributing to military clothing comfort and satisfaction. Textile Research Journal, 75(3), pp.223-232.

Watkins, S.M., 1995. Clothing: The portable environment 2nd ed., Ames, lowa State: University 
Press.

Yoo, I.-G. \& Yoo, W.-G., 2012. Effects of the wearing of tight jeans on lumbar and hip movement during trunk flexion. Journal of Physical Therapy Science, 24(8), pp.659-661. 